\title{
An analysis of the spontaneous mutation rate measurement in filamentous fungi
}

\author{
Marta S. Baracho and Ivanhoé R. Baracho \\ Departamento de Genética e Evolução, Universidade Estudual de Campinas, Campinas, SP, Brazil.
}

\begin{abstract}
Mutations related to gene meth $_{1}$ of Aspergillus nidulans were analyzed, in order to study a mathematical model for the determination of the mutation rate per nucleus per generation, in filamentous fungi. A replica plating technique was used to inoculate, in a single operation, 26 colonies of the strain, into Petri dishes containing culture medium, and the nine central colonies were analyzed for size and number of conidia in each colony. Using this technique, several central colonies were analyzed with regard to the appearance of mutation, and the number and type of reversions were determined for each colony. The frequencies obtained for each reversion were analyzed, in order to verify if their distribution was in accordance with that of Greenwood and Yule. The data obtained allowed us to conclude that, using the mathematical model studied, it is possible to determine the mutation rate per nucleus, per generation, in filamentous fungi.
\end{abstract}

Key words: Aspergillus nidulans, mutation rate, spontaneous mutation, fungi, filamentous fungi.

Received: April 8, 2002; accepted: November 11, 2002.

\section{Introduction}

There are two different situations to be taken into account in the determination of spontaneous mutation rates in microorganisms: determination in organisms that grow as free cells, and determination in organisms that grow as mycelia.

Particularly important studies on the first situation concern bacterial populations subject to mutation. The mathematical aspects of this issue started to be considered of greater importance in 1943, when the report of Luria and Delbruck was published, followed by other mathematical studies on this subject, such as the papers by Shapiro (1946), Lea and Coulson (1949), and Armitage (1952, 1953), which gave rise to the development of various methods for the determination of spontaneous mutation rate in organisms that grow as free cells.

However, in organisms that grow as mycelia, the determination of the spontaneous mutation rate presents some difficulties, and is usually incomplete. The ideal way of doing it would be to determine the mutation probability per nucleus and per generation, as done for bacteria. But, since in filamentous fungi the only easy way of obtaining nucleus samples is through the spores, the usual procedure is to determine the proportion of mutants among the spores

Send correspondence to Marta S. Baracho. E-mail: martbaracho@ hotmail.com. formed, without considering whether the mutation occurred during spore formation or during previous nuclear divisions.

The objective of the present study was to determine if the Greenwood and Yule distribution (1920) is an adequate mathematical model for the determination of spontaneous mutation rate in filamentous fungi.

\section{Materials and Methods}

Media

The minimum medium (MM) used was Czapek-Dox with $1 \%(\mathrm{w} / \mathrm{v})$ glucose. The complete medium (CM) contained yeast extract, hydrolyzed casein, hydrolyzed nucleic acids, vitamins, etc, (Pontecorvo et al., 1953). Solid media contained $2 \%$ agar.

Strain

We used the bi $\mathrm{A}_{1}$, meth $\mathrm{G}_{1}$ strain of Aspergillus nidulans derived from the Glasgow stock, which was maintained on complete medium slants at $5{ }^{\circ} \mathrm{C}$. The genetic markers of this strain are $b i \mathrm{~A}_{1}$ and $m e t h \mathrm{G}_{1}$, which are a requirement, respectively, for biotin and methionine.

\section{Spontaneous mutations}

In order to study the mathematical model, spontaneous mutations related to the gene meth $\mathrm{G}_{1}$ of $A$. nidulans 
were analyzed. In a study on this gene, Lilly (1965) observed that it was a mutation that could be suppressed by specific suppressor genes not linked to the mutant locus. These revertants appeared spontaneously at a frequency of 1 in $6 \times 10^{4}$ conidia, a frequency at least 100 times higher than that observed for other loci. They fall into three visibly distinct types, as follow: type A- colonies which are essentially normal; type B- colonies which produce a brown pigment in the medium and have sparse conidiation; type Cdensely conidiating colonies with hyaline edges.

This methionine system was previously studied by other authors, and different classifications were proposed (Rocha, 1983). However, in the present study the revertants were defined according to the classification of Lilly (1965).

\section{Determination of number of mutants}

The $b i \mathrm{~A}_{1}$, meth $\mathrm{G}_{1}$ strain was inoculated into the center of Petri dishes measuring $9 \mathrm{~cm}$ in diameter containing $20 \mathrm{~mL}$ solid $\mathrm{CM}$, and the preparations were incubated for 9 days at $37^{\circ} \mathrm{C}$. Then, the cultures were replated by means of a 26-needle replicator onto other Petri dishes with $\mathrm{CM}$, and incubated for 4 days. Conidia from each of 9 central colonies were suspended in $1 \mathrm{~mL}$ Tween 80 solution and shaken for disaggregation. The suspension was filtered through cotton wool into test tubes, and the entire content of the test tubes was spread with a Drigalski spatula onto Petri dishes containing minimum medium supplemented with biotin $(0.4 \mu \mathrm{g} / \mathrm{mL})$. The dishes were incubated at $37{ }^{\circ} \mathrm{C}$ for 10 days, and then the number and type of colonies that arose were determined.

\section{Determination of colony size and number of conidia per colony}

Central colonies obtained as described above were measured with a millimeter ruler to determine their diameter and variation. Conidial suspensions obtained from these colonies and treated as described above were counted with a hemocytometer, to estimate the number of conidia per colony.

\section{Determination of conidial viability}

Conidia from colonies allowed to grow in CM for 4 days were placed in Tween 80 solution, disaggregated by shaking, and counted with a hemocytometer. The conidial suspensions were then diluted in saline solution, in order to obtain appropriate numbers for seeding. $0.1 \mathrm{~mL}$ aliquots of this diluted conidial suspension were seeded on Petri dishes containing CM, and spread with a Drigalski spatula. The dishes were incubated for 2 days, and the number of growing colonies was counted.

\section{Mathematical model}

In the present study, we considered Greenwood and Yule's distribution (1920) as the onset model of mutant conidia generated by nuclei that underwent the mutation. These authors, when referring to the number of accidents, assumed that their distribution was of the Poisson type,

$$
f(j)=e^{-\lambda} \frac{\lambda^{j}}{j !}
$$

for $\mathrm{j}=0,1, \ldots$, with $\lambda$ being a random variable which expresses the distinct degrees of individual risk to which the population is subject, and whose distribution function is given by:

$$
\mathrm{dF}=\frac{\mathrm{c}^{\mathrm{p}}}{\Gamma(\mathrm{p})} \mathrm{e}^{-\mathrm{c} \lambda} \lambda^{\mathrm{p}-1} \mathrm{~d} \lambda, \quad \lambda>0 \mathrm{p}>0
$$

with $\mathrm{c}$ and $\mathrm{p}$ being distribution parameters.

Starting from this point, the authors arrived at the development

$$
\left(\frac{\mathrm{c}}{\mathrm{c}+1}\right)^{\mathrm{p}}\left[1, \frac{\mathrm{p}}{\mathrm{c}+1}, \frac{\mathrm{p}(\mathrm{p}+1)}{2 !(\mathrm{c}+1)^{2}}, \ldots\right]
$$

or, in a general form,

$$
f(x)=\left(\frac{\mathrm{c}}{\mathrm{c}+1}\right)^{\mathrm{p}} \cdot \frac{\left|\left(\frac{-\mathrm{p}}{\mathrm{x}}\right)\right|}{(\mathrm{c}+1)^{\mathrm{x}}}
$$

for $\mathrm{x}=0,1$,

The mean $(\mathrm{m})$ and variance $\left(\sigma^{2}\right)$ of this distribution are:

$$
\mathrm{m}=\frac{\mathrm{p}}{\mathrm{c}} \quad \sigma^{2}=\frac{\mathrm{p}}{\mathrm{c}}\left(\frac{\mathrm{c}+1}{\mathrm{c}}\right)
$$

with

$$
c=\frac{m}{\sigma^{2}-m} \quad p=\frac{m^{2}}{\sigma^{2}-m}
$$

Although Greenwood and Yule (1920) studied mainly the occurrence of accidents, considering the different degrees of individual risks, the application of their distribution to the study of mutations proposed here can be easily justified. It is enough to consider the occurrence of mutant conidia in view of the distinct possibilities of the appearance of nuclei where a mutation took place.

\section{Results}

\section{Number of mutants}

Table 1 shows the frequencies of type $\mathrm{A}, \mathrm{B}$, and $\mathrm{C}$ reversions for each colony obtained from strain bi $\mathrm{A}_{1}$, meth $\mathrm{G}_{1}$ by the technique described above.

These data were used to determine the mean number of mutated nuclei, using the Greenwood and Yule distribu- 
Table 1 - Frequencies observed for type A, B, and C reversions.

\begin{tabular}{|c|c|c|c|c|}
\hline \multirow{2}{*}{$\begin{array}{l}\text { Number of } \\
\text { mutants (n) }\end{array}$} & \multicolumn{3}{|c|}{ Number of colonies with $\mathrm{n}$ mutants } & \multirow[b]{2}{*}{ Sum } \\
\hline & A & $\mathrm{B}$ & $\mathrm{C}$ & \\
\hline 0 & & 7 & 9 & 16 \\
\hline 1 & & 4 & 8 & 12 \\
\hline 2 & 1 & 9 & 14 & 24 \\
\hline 3 & 3 & 13 & 11 & 27 \\
\hline 4 & & 9 & 16 & 25 \\
\hline 5 & 4 & 7 & 9 & 20 \\
\hline 6 & 3 & 9 & 6 & 18 \\
\hline 7 & 5 & 7 & 9 & 21 \\
\hline 8 & 6 & 7 & 7 & 20 \\
\hline 9 & 4 & 7 & 1 & 12 \\
\hline 10 & 10 & & 1 & 11 \\
\hline 11 & 9 & 3 & 2 & 14 \\
\hline 12 & 4 & 4 & & 8 \\
\hline 13 & 3 & 1 & & 4 \\
\hline 14 & 6 & 4 & 2 & 12 \\
\hline 15 & 3 & 1 & & 4 \\
\hline 16 & 6 & & & 6 \\
\hline 17 & 8 & 1 & 1 & 10 \\
\hline 18 & 1 & & & 1 \\
\hline 19 & 5 & & & 5 \\
\hline 20 & 2 & & & 2 \\
\hline 22 & 1 & & & 1 \\
\hline 23 & & & 1 & 1 \\
\hline 24 & & 1 & 1 & 2 \\
\hline 25 & 1 & & & 1 \\
\hline 26 & & 1 & 1 & 2 \\
\hline 27 & 1 & & & 1 \\
\hline 28 & 1 & & & 1 \\
\hline Sum & 87 & 95 & 99 & 281 \\
\hline
\end{tabular}

tion. The mean number of nuclei where a mutation took place was obtained by the following formula:

$$
p=\frac{m^{2}}{\sigma^{2}-m}
$$

For type A reversions, we examined 87 colonies, which presented a mean number of 12.30 mutants per colony, and a variance of 28.54. The mean number of nuclei where a mutation took place was 9.31. For type B reversions, we examined 95 colonies, which presented a mean number of 6.17 mutants, and a variance of 23.45. The mean number of nuclei where a mutation took place was 2.21. For type $\mathrm{C}$ reversions, we examined 99 colonies, which presented a mean number of 4.97 mutants per colony, and a variance of 22.34. The mean number of nuclei where a mutation took place was 1.42 .

\section{Conidial viability}

Conidial viability was determined by hemocytometer counting, and the rates obtained were, respectively, $87 \%$ viable conidia for strain bi $\mathrm{A}_{1}$, meth $\mathrm{G}_{1}, 100 \%$ for the type $\mathrm{A}$ reversion, $83 \%$ for the type $\mathrm{B}$ reversion, and $71 \%$ for type $\mathrm{C}$ reversions.

\section{Colony size and number of conidia per colony}

Fifty-four central colonies of the strain biA1, meth $\mathrm{G} 1$ obtained as described earlier were measured, and their diameter was found to range from 11 to $14 \mathrm{~mm}$, with a mean and standard error of the mean of $11.89 \pm 0.12$. The number of conidia of these 9 colonies was estimated by hemocytometer counting, and the mean detected was $3.8 \mathrm{x}$ $10^{7} \pm 6.1 \times 10^{6}$ conidia per colony.

\section{Discussion}

\section{Frequency of mutants}

Three types of phenotypically distinguishable reversions of the meth $\mathrm{G}_{1}$ gene were studied. The number of mutants detected in each colony is listed in Table 1.

Several studies using the same strain (originated in Glasgow, UK) to detect the mutagenic activity of physical and chemical agents have reported results referring to these spontaneous reversions. Siddiqui (1962) reported a spontaneous mutation frequency of about $8.2 \times 10^{-6}$, with $34.2 \%$ for type A, $31.2 \%$ for type B, and $35 \%$ for Type C, respectively.

Lilly (1965) reported a frequency of 1 in $6 \times 10^{4}$, which is approximately $17 \times 10^{-6}$, with rates of $66 \%$ for Type A, 31\% for type B, and 3\% for Type C, respectively. Alderson and Clark (1966) conducted two experiments. In one of them, they detected frequencies of $9.2 \times 10^{-6}$, with a distribution of $35 \%$ for Type A, $65 \%$ for Type B, and $0 \%$ for Type $\mathrm{C}$. In the other experiment, the frequency was $1.1 \times 10^{-6}$, with a distribution of $63 \%$ for Type A, $29 \%$ for Type B, and $8 \%$ for Type C. In contrast, data reported by Duarte (1972) showed a frequency of $12.6 \times 10^{-6}$, with a distribution of $58 \%$ for Type A, 31\% for Type B, and $11 \%$ for Type C. Scott et al. (1973) reported frequencies of $2 \times 10^{-6}$ to $5 \times 10^{-6}$, while Rocha (1983) detected variations from $6.4 \times 10^{-6}$ to $20.4 \times 10^{-6}$.

In the present study, we detected a frequency of $0.95 \times 10^{-6}$ (approximately $1 \times 10^{-6}$ ), with a proportion of $61 \%$ for Type A, 25\% for Type B, and 14\% for Type C.

Thus, the data reported by the various authors are discrepant. However, in the present study we determined the number of mutants per colony and estimated the number of conidia in each colony, whereas in the other studies the 
number of conidia was estimated in a suspension, and a known volume of this suspension was used to determine the number of mutants that arose.

\section{Mean number of nuclei that underwent a mutation}

The frequency of mutant conidia does not provide an actual idea of the number of mutations that actually occurred, since, in the case of Aspergillus nidulans, the conidia are formed from secondary sterigmas by repeated nuclear division. The conidia start to develop as cytoplasmic protuberances at the end of the sterigmas. The nucleus of the sterigma then undergoes one mitotic division and, while one of the two resulting nuclei enters the region that will be transformed into a conidium, the other moves to the opposite end. As the conidium becomes delimited, the nucleus of the sterigma starts to divide again, to produce another conidium. The process thus continues, with the formation of a long chain in which the last conidium formed is closest to the sterigma, while the conidium that was first formed is the most distant one (Timberlake, 1990).

In view of this formation process, it is clear that the number of mutant conidia in a chain will depend on the moment when the mutation occurred in the nucleus of the sterigma. If the mutation occurred long before sterigma formation, obviously an entire conidial head may be mutated. Therefore, the frequency of mutant conidia does not correspond to the frequency of mutations that actually occurred per nucleus, and consequently the mutation rate cannot be determined on this basis.

As pointed out by Fincham and Day (1971), the ideal in this case would be "to measure the chance of mutation occurring per nucleus per time unit, or per nuclear generation time." This is possible in organisms that grow as free cells. In filamentous fungi, however, a nucleus sample is easily obtained only by using spores, which makes it difficult to determine the mutation rate. Usually, what is determined is the frequency of mutating spores.

This is what is commonly done in mutation studies with the $b i \mathrm{~A}_{1}$ meth $\mathrm{G}_{1}$ strain. In this case, the nucleus samples come from conidial samples in which, as mentioned earlier, the number of mutant nuclei does not correspond to the number of nuclei that underwent mutation. Therefore, a mathematical model has to be established that permits to estimate the number of nuclei that underwent mutation based on the number of mutant conidia, like the distribution of Greenwood and Yule.

\section{The model}

Frequencies were calculated for a theoretical distribution (Greenwood and Yule distribution) and for the sampling data referring to Type $\mathrm{A}, \mathrm{B}$, and $\mathrm{C}$ reversions, and an adherence test was performed to determine whether the fit of the data observed to the theoretical distribution chosen was good. The adherence test used was the Kolmogorov-Smirnov test, used to determine whether the observed accumulated frequency departs significantly from a hypothesized frequency distribution. The point where these two distributions - theoretical and observed - show the greatest divergence is determined, and this maximum observed deviation (D) is compared to a tabled value, to determine if it is too large to be attributed to chance (Siegel, 1975).

Thus, the Kolmogorov-Smirnov test can demonstrate if the mathematical model chosen serves the purpose of the study, which in our case was the capacity to estimate the mean number of nuclei that underwent mutation based on the number of mutant conidia, with a consequent estimate of the mutation rate per nucleus per generation.

The result of the Kolmogorov-Smirnov test for Type A reversions can be seen in Table 2. A D value of 0.0444 , non-significant at the $5 \%$ level of probability, was detected, thus indicating that the Greenwood and Yule distribution fits the data. The results concerning Type B reversions are listed in Table 3. In this case, the value of D was 0.0361 , also non-significant at the $5 \%$ level of probability. For Type $\mathrm{C}$ reversions, the results are presented in Table 4, which shows a value of $\mathrm{D}=0.0877$, non-significant at the $5 \%$ level as well.

Table 2 - Determination of the goodness of fit of the frequencies observed for type A reversions (Table 1) to the theoretical frequencies of the Greenwood-Yule distribution (Kolmogorov-Smirnov test).

\begin{tabular}{|c|c|c|c|c|c|}
\hline $\begin{array}{l}\text { Number of } \\
\text { mutants }\end{array}$ & $\begin{array}{l}\text { Observed } \\
\text { frequency }\end{array}$ & $\begin{array}{l}\text { Expected } \\
\text { frequency }\end{array}$ & $\begin{array}{l}\text { a-accumul } \\
\text { ated obs. } \\
\text { frequency }\end{array}$ & $\begin{array}{l}\text { b-accumul } \\
\text { ated exp. } \\
\text { frequency }\end{array}$ & $\begin{array}{l}\text { Difference } \\
(\mathrm{D}=\mathrm{b}-\mathrm{a})\end{array}$ \\
\hline 0 & 0 & 0.004 & 0 & 0.004 & 0.004 \\
\hline 1 & 0 & 0.0021 & 0 & 0.0025 & 0.0025 \\
\hline 2 & 0.0115 & 0.0061 & 0.0115 & 0.0086 & -0.0029 \\
\hline 3 & 0.0345 & 0.0131 & 0.046 & 0.0217 & -0.0217 \\
\hline 4 & 0 & 0.023 & 0.046 & 0.0447 & -0.0013 \\
\hline 5 & 0.0459 & 0.0348 & 0.0919 & 0.0795 & -0.0124 \\
\hline 6 & 0.0345 & 0.0472 & 0.1264 & 0.1267 & 0.0003 \\
\hline 7 & 0.0575 & 0.0587 & 0.1839 & 0.1854 & 0.0015 \\
\hline 8 & 0.0689 & 0.0681 & 0.2528 & 0.2535 & 0.0007 \\
\hline 9 & 0.0459 & 0.0745 & 0.2987 & 0.328 & 0.0293 \\
\hline 10 & 0.1149 & 0.0775 & 0.4136 & 0.4055 & -0.0081 \\
\hline 11 & 0.1034 & 0.0774 & 0.517 & 0.4829 & -0.0341 \\
\hline 12 & 0.0459 & 0.0745 & 0.5629 & 0.5574 & -0.0055 \\
\hline 13 & 0.0345 & 0.0694 & 0.5974 & 0.6268 & -0.0294 \\
\hline 14 & 0.0689 & 0.0629 & 0.6663 & 0.6897 & 0.0234 \\
\hline 15 & 0.0345 & 0.0555 & 0.7008 & 0.7452 & $0.0444 *$ \\
\hline 16 & 0.0685 & 0.048 & 0.7693 & 0.7932 & 0.0239 \\
\hline 17 & 0.0919 & 0.041 & 0.8612 & 0.8342 & -0.027 \\
\hline 18 & 0.0115 & 0.0341 & 0.8727 & 0.8683 & -0.0044 \\
\hline 19 & 0.0575 & 0.0278 & 0.9302 & 0.8961 & -0.0341 \\
\hline 20 & 0.023 & 0.0244 & 0.9532 & 0.9206 & -0.0326 \\
\hline
\end{tabular}

$* D=0.0444-$ Non-significant at the $5 \%$ level of probability. 
Table 3 - Determination of the goodness of fit of the frequencies observed for type B reversions (Table 1) to the theoretical frequencies of the Greenwood-Yule distribution (Kolmogorov-Smirnov test).

\begin{tabular}{lllccc}
\hline $\begin{array}{l}\text { Number of } \\
\text { mutants }\end{array}$ & $\begin{array}{c}\text { Observed } \\
\text { frequency }\end{array}$ & $\begin{array}{c}\text { Expected } \\
\text { frequency }\end{array}$ & $\begin{array}{c}\text { a-accumul } \\
\text { ated obs. } \\
\text { frequency }\end{array}$ & $\begin{array}{c}\text { b-accumul } \\
\text { ated exp. } \\
\text { frequency }\end{array}$ & $\begin{array}{c}\text { Difference } \\
(\mathrm{D}=\mathrm{b}-\mathrm{a})\end{array}$ \\
\hline 0 & 0.0737 & 0.0527 & 0.0737 & 0.0527 & -0.021 \\
1 & 0.0421 & 0.0857 & 0.1158 & 0.1384 & 0.023 \\
2 & 0.0947 & 0.1011 & 0.2105 & 0.2395 & 0.029 \\
3 & 0.1368 & 0.1044 & 0.3473 & 0.3439 & -0.0034 \\
4 & 0.0947 & 0.1002 & 0.442 & 0.4441 & -0.0021 \\
5 & 0.0737 & 0.0913 & 0.5157 & 0.5356 & 0.0199 \\
6 & 0.0947 & 0.081 & 0.6104 & 0.6166 & -0.0062 \\
7 & 0.0737 & 0.07 & 0.6841 & 0.6866 & 0.0025 \\
8 & 0.0737 & 0.0594 & 0.7578 & 0.7459 & -0.0119 \\
9 & 0.0737 & 0.0495 & 0.8315 & 0.7954 & $-0.0361 *$ \\
10 & 0 & 0.0409 & 0.8315 & 0.8363 & 0.0048 \\
11 & 0.0316 & 0.0334 & 0.8631 & 0.8697 & 0.0066 \\
12 & 0.0421 & 0.0271 & 0.9052 & 0.8968 & -0.084 \\
13 & 0.0105 & 0.0218 & 0.9157 & 0.9186 & 0.0029 \\
14 & 0.0421 & 0.0174 & 0.9578 & 0.936 & -0.0218 \\
15 & 0.0105 & 0.0128 & 0.9683 & 0.9488 & -0.0195 \\
16 & 0 & 0.011 & 0.9683 & 0.9598 & -0.0085 \\
17 & 0.0105 & 0.0087 & 0.9783 & 0.9685 & -0.0098 \\
18 & 0 & 0.0068 & 0.9788 & 0.9753 & -0.0035 \\
19 & 0 & 0.0053 & 0.9788 & 0.9806 & 0.0018 \\
20 & 0 & 0.0042 & 0.9788 & 0.9848 & 0.006 \\
\hline & & & & &
\end{tabular}

$* \mathrm{D}=0,0361-$ Non-significant at the $5 \%$ level of probability.

Table 4 - Determination of the goodness of fit of the frequencies observed for type $\mathrm{C}$ reversions (Table 1) to the theoretical frequencies of the Greenwood-Yule distribution (Kolmogorov-Smirnov test).

\begin{tabular}{lllccc}
\hline $\begin{array}{l}\text { Number of } \\
\text { mutants }\end{array}$ & $\begin{array}{c}\text { Observed } \\
\text { frequency }\end{array}$ & $\begin{array}{c}\text { Expected } \\
\text { frequency }\end{array}$ & $\begin{array}{c}\text { a-accumul } \\
\text { ated obs. } \\
\text { frequency }\end{array}$ & $\begin{array}{c}\text { b-accumul } \\
\text { ated exp. } \\
\text { frequency }\end{array}$ & $\begin{array}{c}\text { Difference } \\
(\mathrm{D}=\mathrm{b}-\mathrm{a})\end{array}$ \\
\hline 0 & 0.0909 & 0.1179 & 0.0909 & 0.1179 & 0.027 \\
1 & 0.0808 & 0.1304 & 0.1717 & 0.2484 & 0.0767 \\
2 & 0.1414 & 0.1228 & 0.3131 & 0.3712 & 0.0581 \\
3 & 0.1111 & 0.1033 & 0.4242 & 0.4746 & 0.0504 \\
4 & 0.1616 & 0.0936 & 0.5858 & 0.5682 & -0.0176 \\
5 & 0.0909 & 0.0789 & 0.6767 & 0.6472 & 0.0295 \\
6 & 0.0606 & 0.0656 & 0.7373 & 0.7129 & -0.0245 \\
7 & 0.0909 & 0.0541 & 0.8282 & 0.7669 & -0.0613 \\
8 & 0.0707 & 0.0443 & 0.8989 & 0.8112 & $-0.0877 *$ \\
9 & 0.0101 & 0.0360 & 0.909 & 0.8472 & -0.0618 \\
10 & 0.0101 & 0.0292 & 0.9191 & 0.8764 & -0.0427 \\
11 & 0.0202 & 0.0236 & 0.9393 & 0.9 & -0.0393 \\
12 & 0 & 0.0190 & 0.9393 & 0.9119 & -0.0274 \\
13 & 0 & 0.0152 & 0.9393 & 0.9271 & 0.0122 \\
14 & 0.0202 & 0.0122 & 0.9595 & 0.9393 & 0.0202 \\
15 & 0 & 0.0098 & 0.9595 & 0.9491 & 0.0104 \\
16 & 0 & 0.0078 & 0.9595 & 0.9569 & 0.0026 \\
17 & 0.0101 & 0.0062 & 0.9696 & 0.9631 & 0.0065 \\
18 & 0 & 0.0049 & 0.9696 & 0.968 & 0.0016 \\
19 & 0 & 0.0039 & 0.9696 & 0.9719 & 0.0023 \\
20 & 0 & 0.0031 & 0.9696 & 0.975 & 0.0054 \\
\hline
\end{tabular}

$* \mathrm{D}=0.0877$ - Non-significant at the $5 \%$ level of probability.
The sampling frequencies of the three types of reversions, therefore, fit the theoretical frequencies of Greenwood and Yule's distribution. Consequently, it is valid to use this distribution to calculate the mean number of mutated nuclei based on the number of mutant conidia. This estimate definitely permits the calculation of the mutation rate per nucleus per generation, when the data concern a single gene.

\section{Acknowledgments}

This work was supported by Capes.

\section{References}

Alderson T and Clark AM (1966) Interlocus specificity for chemical mutagens in Aspergillus nidulans. Nature 210:593-595.

Armitage P (1952). The statistical theory of bacterial populations subject to mutation. Journal of Royal Statistical Society $14: 1$.

Armitage P (1953) Statistical concepts in the theory of bacterial mutation. Journal of Hygiene 51:162-183.

Duarte FM (1971) Efeitos mutagênicos de alguns ésteres de ácidos inorgânicos em Aspergillus nidulans. Ciência e Cultura 24:42-52.

Fincham JRS and Day PR (1971) Fungal Genetics. Botanical Monographs, v. 4, Blackwell Scientific Publications. Philadelphia, Pennsylvania.

Greenwood M and Yule GU (1920) An enquiry into the nature of frequency distributions representative of multiple happenings with particular reference of multiple attacks of disease or of repeated accidents. Journal Royal Statistical Society $83: 255-279$

Lea DE and Coulson CA (1949) The distribution of the numbers of mutants in bacterial populations. Journal of Genetics 49:264-285.

Lilly JL (1965) An investigation of the suitability of the suppressors of meth1 in Aspergillus nidulans for study of induced and spontaneous mutation. Mutation Research 2:192-195.

Luria SE and Delbruck M (1943) Mutations of bacteria from virus sensitivity to virus resistance. Genetics 28:491-511.

Pontecorvo G, Roper JA, Hemmons LM, MacDonald KD and Bufton AW (1953) The genetics of Aspergillus nidulans. Advances in Genetics 5:141-238.

Rocha CLMSC (1983) Detecção da atividade mutagênica de produtos químicos no sistema meth1 em $A$. nidulans. Tese de Mestrado, pp 77.

Scott BR, Alderson T and Papworth DG (1973) The effect of plating densities on the retrieval of methionine suppressor mutation after ultraviolet or gamma irradiation of Aspergillus. Journal of General Microbiology 75:235-239.

Shapiro A (1946) The kinetics of growth and mutation in bacteria. Cold Spring Harbor Symposia on Quantitative Biology 11:228.

Siddiqui OH (1962) Mutagenic action of nitrous acid on Aspergillus nidulans. Genetical Research 3:303-314.

Siegel S (1975) Estatística não-paramétrica. Editora Mcgraw-Hill do Brasil, São Paulo, pp 332.

Timberlake WE (1990) Molecular genetics of Aspergillus development. Annual Review Genetics 24:5-36. 